\title{
Estudio de la ansiedad y el estrés en personas con trastorno del espectro del autismo en los procesos de inclusión laboral
}

\section{Montserrat Clavero Padrós}

Directora del área clínica, APNABI-Autismo Bizkaia

\section{Iban Huarte Esteban}

Director del área sociolaboral, APNABI-Autismo Bizkaia

\section{Leire Redondo Urbieta}

Psicóloga del área sociolaboral, APNABI-Autismo Bizkaia

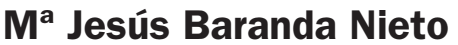

Psicóloga del área clínica, APNABI-Autismo Bizkaia

\section{$M^{\mathbf{a}}$ Luisa Arenas Ortega}

Técnica de apoyo laboral del área sociolaboral, APNABI-Autismo Bizkaia marialuisa.arenas@entremanos.eus

Autismoaren espektroaren nahasmendua antsietateeta estres-maila handiekin bat gertatutako egoera edo nahasmendua izaten da. Antsietatea giltzarrietako bat da autismoaren espektroaren nahasmendua duten pertsona askoren bizitzan, eta kontuan hartu behar da bizi diren testuinguruetan, hala nola, laneratzeko prozesuetan. Sarritan, bilaketa- eta laneratze-prozesuek eragiten duten antsietatea eta estresa oztopo izan ohi dira pertsona horien bizi-plan propioak lortzeko. Artikulu honetan, eremu soziolaboraleko estres- eta antsietatemailen ebaluazioaren hasierako emaitzak aurkezten dira, baita APNABI-Autismo Bizkaia elkartearen erabiltzaileak diren autismoaren espektroaren nahasmendua duten pertsonen eragina ere, autismorik gabeko pertsonak kontrastatzeko talde batekin alderatuta. Ikerketa Eusko Jaurlaritzaren proiektu berezien baitakoa da.

\section{GAKO-HITZAK:}

Autismoaren espektroaren nahasmendua, antsietatea, estres soziolaborala, enplegua.
El trastorno del espectro del autismo (TEA) es una condición o trastorno que suele coocurrir con niveles elevados de ansiedad y estrés. La ansiedad es una piedra angular en la vida de muchas personas con TEA, y se ha de tener en cuenta en los diferentes contextos donde se desenvuelven, como, por ejemplo, en los procesos de inclusión laboral. Con frecuencia la ansiedad y el estrés que provocan los procesos de búsqueda y de inserción laboral pueden ser un freno para que estas personas logren sus planes de vida. Este artículo presenta los resultados iniciales de la evaluación de los niveles de estrés y ansiedad en el ámbito sociolaboral y cómo influyen en las personas con TEA, usuarias de la Asociación APNABI-Autismo Bizkaia, en comparación con un grupo de contraste de personas sin TEA. El estudio se enmarca dentro de los Proyectos Singulares del Gobierno Vasco.

\section{Palabras Clave:}

Trastorno del espectro del autismo, ansiedad, estrés sociolaboral, empleo. 


\section{Introducción}

El trastorno del espectro del autismo (TEA) o la condición de espectro del autismo (CEA), como viene considerándose recientemente, es un trastorno del neurodesarrollo (TND) que supone la presencia de dificultades en la comunicación e interacción social, así como conductas e intereses restringidos o repetitivos (American Psychiatric Association, 2013). El objetivo de este artículo es poder poner de manifiesto una realidad mostrada por las personas adultas diagnosticadas de esta condición, con las que se trabaja desde el área sociolaboral de la Asociación de familias de personas afectadas de trastorno del espectro del autismo de Bizkaia (APNABI-Autismo Bizkaia).

Dentro de APNABI-Autismo Bizkaia se apuesta por el desarrollo de programas que contribuyan a proporcionar apoyos y oportunidades a la personas con TEA, para promover su inclusión social como ciudadanos de pleno derecho. Desde el área sociolaboral, se viene observando que una de las principales dificultades manifiestas en las personas adultas con TEA, especialmente acusada en el momento de búsqueda de empleo e inserción laboral, son los elevados niveles de estrés y ansiedad que con frecuencia soportan. Cuando se enfrentan a esta circunstancia, desde el equipo de profesionales se ha constatado que se disparan sus respuestas de ansiedad ante un acontecimiento que, si bien podemos considerar estresante para el resto de la población, a las personas con TEA les suele dificultar mucho su vida cotidiana.

Ante esta circunstancia, se plantea el desarrollo de un estudio que permita refrendar si esta percepción de los/as profesionales concuerda con la percepción de las personas con TEA. Asimismo, se pretende que una vez detectada y cuantificada esta situación, se puedan definir posibles herramientas que les ayuden en su manejo y contribuyan a su bienestar.

Gracias a las ayudas del Gobierno Vasco para la realización de proyectos singulares, se ha puesto en marcha este proyecto, el cual plantea poder conocer los niveles de estrés y ansiedad que manifiestan las personas adultas con TEA en los procesos de búsqueda de empleo y/o inclusión laboral en el mercado ordinario de trabajo. Asimismo, ofrece una formación a las personas participantes dirigida a la adquisición de herramientas que les faciliten el afrontamiento de dichos procesos de la mejor manera posible, reconociendo y manejando la ansiedad y el estrés que les pueda producir. De todo ello, se obtendrán las conclusiones del estudio. Con él se pretende poner de relieve la importancia de reconocer e investigar con mayor profundidad estos estados en las personas con TEA, lo que permitirá el desarrollo de recursos y programas de intervención que contribuyan a mejorar su calidad de vida.

El estudio que se inició a finales del 2019, se ha visto frenado por la situación actual provocada por la alerta sanitaria de la covid-19 y, por lo tanto, lo que se presenta en este artículo es únicamente lo que hasta la fecha se ha podido desarrollar. Sin embargo, conscientes de que se enmarca en un proyecto aún por terminar, se considera que tanto el planteamiento de base como los resultados preliminares alcanzados pueden resultar de gran interés para otros/as profesionales.

La estructura de este documento se conforma, en primer lugar, por el fundamento teórico relacionado con el TEA y la ansiedad, así como los objetivos e hipótesis de trabajo que se plantean. En segundo lugar, se describe la metodología utilizada (procedimiento, participantes, instrumentos y análisis estadísticos) y, finalmente, se concluye con una discusión acerca de los resultados preliminares obtenidos.

\section{Fundamento teórico: ansiedad, estrés y TEA}

La ansiedad es una condición universal, ya que se basa en la emoción de miedo y surge como una respuesta ante estímulos evaluados como amenazantes. El miedo es una emoción que permite preservar la integridad de las personas, puesto que se desencadena ante un estímulo potencialmente dañino e incita a los individuos a huir o luchar contra dicho estímulo. Por su parte, el estrés es una reacción inmediata e intensa, que implica la movilización de recursos del organismo (fisiólogicos, cognitivos y motores) y que se produce ante situaciones que suponen importantes demandas para el individuo, ya sea porque implican una pérdida, o por tratarse de una amenaza o de un reto (Crespo y Labrador, 2003). Se considera una respuesta adaptativa que prepara al organismo para actuar y enfrentar tales situaciones, pero que aparece ante un estresor externo e identificable. Ante la desaparición de este, las respuestas desencadenadas cesan.

Las similitudes entre ambas respuestas hacen que con frecuencia los términos estrés y ansiedad se utilicen indistintamente. Sin embargo, podríamos señalar que mientras que el estrés se genera por un evento o situación externa concreto, los factores que desencadenan la ansiedad suelen ser más difusos y con frecuencia el origen se encuentra no tanto en la demanda del medio, sino en la interpretación y/o preocupación de la persona ante una situación amenazante presente o futura.

La ansiedad aparece, por tanto, ante la anticipación de una potencial amenaza, e involucra respuestas de corte conductual, cognitivo, fisiológico y afectivo (Clark y Beck, 2012). Sin embargo, esta ansiedad adaptativa puede llegar a convertirse en dañina si se prolonga en el tiempo, o si es más intensa o más frecuente de lo esperado, es decir, si se cronifica, provocando una reacción más exagerada de lo que cabría esperar para esa situación, o bien si aparece ante un número elevado de acontecimientos. De 
este modo, se apuesta por el pensamiento como variable mediadora entre el estímulo y la respuesta $\mathrm{y}$, por tanto, las distorsiones cognitivas influyen en la importancia que se le otorga a cada situación. Además, para considerar si esa ansiedad es desadaptativa para la persona, cabe valorar sus estrategias de coping 0 afrontamiento, pues si estas son inadecuadas o deficientes influirán en la aparición o mantenimiento de dicha ansiedad disfuncional, que puede incluso llegar a ser clínicamente significativa (Paula, 2015).

El TEA es un trastorno estrechamente ligado a altos niveles de ansiedad, dado a su elevada coocurrencia. Este punto genera controversia, pues se desconoce si la sintomatología ansiosa es comórbida y, por tanto, fenotípica y etiológicamente idéntica a la que aparece en personas neurotípicas, o si, por el contrario, puede relacionarse con las características propias del TEA, apareciendo de manera diferente en función de la persona y pudiendo dar lugar a distintos perfiles dentro del espectro. También cabe la posibilidad de que los diagnósticos relativos a los trastornos de ansiedad en estas personas sean poco precisos, debido a las dificultades para distinguir signos y síntomas, y realizar un adecuado diagnóstico diferencial (Paula, 2012; Postorino, Kerns, Vivanti, Bradshaw, Siracusano y Mazzone, 2017). Lo que sí se puede asumir es que no todas las personas con TEA sufren de una ansiedad clínicamente significativa (Paula, 2015; Sánchez, Alonso y Alcantud, 2018), por lo que no se debería generalizar la ansiedad como un componente patognomónico de TEA o, incluso, concebir el TEA como un trastorno de estrés, tal y como apuntan algunos autores (Morgan, 2006, citado en Paula, 2015).

Una reciente investigación indica la presencia de tres variables transdiagnósticas, la intolerancia a la incertidumbre, la disfunción ejecutiva y la desregulación emocional, que están en la base tanto de los trastornos de ansiedad como del TEA. Estas variables actúan como mediadoras de la aparición de la sintomatología ansiosa, siempre teniendo en cuenta que existen ciertos factores de riesgo previos, tanto propios al TEA como compartidos con la población neurotípica. Este modelo de transdiagnóstico, que se abordará con mayor precisión más adelante, aboga por una perspectiva integradora y dimensional, donde ambas condiciones comparten ciertas características fisiológicas, conductuales, cognitivas y neurobiológicas (Beneytez, 2019).

Tomando como referencia los datos de coocurrencia de ambas categorías, tal y como están definidas en los actuales manuales nosológicos, los estudios apuntan porcentajes que giran en torno al $11 \%$ y el $84 \%$ (Van Steensel, Bógels y Perrin, 2011, citados en Paula, 2015). Un meta-análisis de 31 estudios acerca de la ansiedad en los jóvenes con TEA menores de 18 años reveló que en torno a un $39,6 \%$ de ellos presentan niveles clínicamente elevados de ansiedad, o incluso el diagnóstico de algún trastorno de ansiedad. Con mayor frecuencia, se encuentra la fobia social (30\%), seguida del trastorno obsesivo compulsivo (TOC) (17\%), la agorafobia (17\%), la ansiedad generalizada (15\%), la ansiedad de separación (9\%) y, finalmente, las crisis de angustia (2\%) (Van Steensel, Bögels y Perrin, 2011). Por tanto, puede destacarse que la ansiedad es un factor relevante en población con TEA, ya que su prevalencia supera las tasas encontradas en población infantil -15\%- (Beesdo, Knappe y Pine, 2009, citados en Paula, 2015) y adulta, sin TEA -18\%- (Kessler, Chiu, Demler y Walters, 2005, citados en Paula, 2015).

\subsection{Hipótesis explicativas de la vulnerabilidad a la ansiedad en TEA}

Según Paula (2013), se plantean dos hipótesis explicativas de dicha coocurrencia: hipótesis del "error social" e hipótesis de la "carga alostática". Con relación a la primera de las hipótesis, el planteamiento es el siguiente: las personas con TEA normalmente tienen experiencias sociales negativas debidas a las dificultades que presentan para integrar la información que proviene del mundo social. Esa carencia a la hora de intuir, inferir, interpretar los pensamientos, sentimientos y conductas de otras personas, e incluso las propias, se ha relacionado principalmente con su déficit en teoría de la mente (ToM), su alexitimia y su alteración en la pragmática del lenguaje (Paula, 2015). La ToM se configura como un constructo que se encuentra esencialmente alterado en personas con TEA (Baron-Cohen, Leslie y Frith, 1985, citados en TirapuUstárroz et al., 2007). Supone la capacidad para intuir, predecir e inferir el comportamiento ajeno, sus creencias, su pensamiento y sus sentimientos (Tirapu-Ustárroz et al., 2007). Por otro lado, la alexitimia hace referencia a la ausencia de palabras para expresar emociones, de modo que las personas con TEA reflejan una falta de reconocimiento emocional tanto propio como de terceros, junto con una escasa capacidad imaginativa (Paula, 2015). Por último, la pragmática del lenguaje se configura como la capacidad para inferir lo que no está explícito en la comunicación, es decir, ir más allá de la literalidad del lenguaje e interpretar las intenciones del interlocutor (Ivern, 2017; Paula, 2015), capacidad que tienen comprometida estas personas por las características propias de su trastorno (American Psychiatric Association, 2013).

Las personas con TEA de "alto funcionamiento" tienden a reflejar mayores niveles de ansiedad, mediados por los problemas que tienen en el ámbito social. Suelen presentar expectativas de corte negativo relacionadas con las experiencias sociales, es decir, esperan un fracaso social que las angustia, ya que desearían adaptarse a las exigencias psicosociales de su entorno. Por tanto, esa autovaloración negativa desemboca en un miedo a sentirse juzgadas que da lugar a la reducción de experiencias sociales y, por tanto, la consolidación 
de ese pensamiento negativo y limitante a nivel social (Hervás, 2016; Paula, 2013).

La segunda hipótesis que plantea Paula (2013) hace referencia a la "carga alostática", es decir, el cúmulo de experiencias negativas, frustrantes, de desconcierto o confusión, que derivan en una carga ansiógena para ellos. Las personas con TEA encuentran dificultades para interpretar las señales sociales de su entorno y ajustarse al contexto. Por ello, como se ha comentado anteriormente, tienden a percibir más situaciones como amenazantes, y esa hiperactivación de su sistema de respuesta ante el estrés, deriva en un estado de mayor alteración fisiológica basal frente a la población neurotípica. El circuito neurobiológico implicado en este fenómeno estaría formado, principalmente, por la amígdala, el córtex prefrontal medial y el hipocampo, actuando estos como un neurocircuito conectado (Kim, Loucks, Palmer, Brown, Solomon y Cois, 2011, citados en Paula, 2015). En las personas con TEA, estas estructuras neuroanatómicas, sobre todo la amígdala y el hipocampo, sufren alteraciones en su crecimiento, observándose en la mayoría de los estudios un tamaño superior en personas con TEA frente a individuos neurotípicos, aunque los resultados no son concluyentes (Paula, 2015).

A modo de inferencia, estas hipótesis se podrían situar dentro del marco teórico de los modelos explicativos de corte cognitivo, donde los sesgos en el procesamiento de la información condicionan la aparición de la sintomatología ansiosa, así como los modelos centrados en el déficit de regulación emocional (Beneytez, 2019). A su vez, cabe tener en cuenta el modelo de intolerancia a la incertidumbre, que parte de un procesamiento erróneo donde estímulos neutros son valorados como amenazantes (Carleton, 2012, citado en Beneytez, 2019), y el de la disfunción ejecutiva, donde entran en juego factores tan relevantes como la flexibilidad cognitiva y el control atencional, que tienden a presentar desajustes (Beneytez, 2019). Estos cuatro modelos son los que tradicionalmente se han manejado para explicar la presencia de estados ansiosos en las personas.

\subsection{Hipótesis explicativas de la ansiedad en TEA a partir de modelos transdiagnósticos}

Los componentes extraídos de los modelos señalados anteriormente (intolerancia a la incertidumbre, desregulación afectiva y disfunción ejecutiva), pueden configurarse como dimensiones comunes tanto para el TEA como para los trastornos de ansiedad, desde una mirada transdiagnóstica. Esta perspectiva aboga por eliminar la categorización de estas condiciones, y ampliar la visión más allá de la dicotomía de síntomas y signos que configuran una determinada etiqueta diagnóstica (Beneytez, 2019). Cabe mencionar dos modelos transdiagnósticos: "modelo de desarrollo y mantenimiento de los trastornos de ansiedad"
(Norton y Paulus, 2017, citados en Beneytez, 2019) y "modelo transdiagnóstico de la triple vulnerabilidad" (Barlow, 2000, citado en Beneytez, 2019). El primero defiende la existencia de una vulnerabilidad biológica y neurológica, en combinación con una de corte psicológico que se ha ido desarrollando desde edades tempranas. Según su planteamiento, la ansiedad se perpetúa debido a las estrategias usadas para mantener y reforzar las creencias irracionales que surgen en relación con la amenaza (Norton y Paulus, 2017, citados en Beneytez, 2019). Por su parte, el segundo modelo hace alusión a una vulnerabilidad biológica de predisposición genética o biológica, junto a una vulnerabilidad psicológica primaria, influida por las experiencias de la infancia, y otra secundaria, mediada por las experiencias del paso de la vida (Barlow, Ellard, Sauer-Zavala, Bullis y Carl, 2014, citados en Beneytez, 2019).

En el caso de las personas con TEA, es probable que la vulnerabilidad biológica que presentan venga de la mano de su trastorno, es decir, de esa alteración que sufren en el neurodesarrollo, junto con vulnerabilidades más específicas relacionadas con su disfunción ejecutiva, su intolerancia a la incertidumbre y su desregulación emocional (Beneytez, 2019). En concreto, se puede señalar que la ansiedad en estas personas se engloba dentro de cuatro dimensiones: la ansiedad de ejecución, que está relacionada con el miedo a fallar en el contexto social y académico; la ansiedad ante la incertidumbre, vinculada con el miedo que les producen los imprevistos, es decir, aquello que no pueden anticipar; la ansiedad de separación asociada al miedo por desprenderse de sus figuras de referencia; y, finalmente, el arousal ansioso, como sintomatología fisiológica que indica que el grado de activación es elevado (Beneytez, 2019).

\subsection{Evaluación de la ansiedad en TEA}

Las personas con TEA tienden a valorar sus niveles de ansiedad como inferiores a lo que reportan sus propios familiares, a pesar de las evidencias científicas a favor de unos niveles de ansiedad superiores por parte de esta población clínica. Por tanto, sus dificultades para reconocer emociones, expresarlas a través de palabras e identificar la intensidad con la que sienten cada emoción, pueden interferir en la autoevaluación de su ansiedad (Paula, 2013). A su vez, otras dificultades como el solapamiento de síntomas entre ambas condiciones o la manifestación poco común de los síntomas de ansiedad, limita el proceso de evaluación de la ansiedad en estas personas (Beneytez, 2019). A día de hoy no existen instrumentos específicos para la evaluación de la ansiedad en personas con TEA, sino que se tienden a aplicar los que se usan para población sin TEA, con las limitaciones que ello conlleva, si bien, para lograr una mayor precisión en dicha evaluación, se suelen incluir apoyos visuales que facilitan a la persona con TEA la comprensión de los estados emocionales y los niveles o grados 
de intensidad de las emociones. Algunas de estas herramientas visuales pueden ser: semáforos de colores, termómetros de emociones o escalas graduadas con números (Paula, 2015).

\subsection{Intervención sobre la ansiedad en TEA}

El diseño de la intervención en personas con TEA que padecen de sintomatología ansiosa debe programarse contemplando las variables anteriormente citadas, sobre todo la intolerancia a la incertidumbre y la atipicidad sensorial (Beneytez, 2019). Podrían plantearse varios niveles de actuación dentro de un programa individualizado de atención a estas personas. El primer nivel implicaría la modificación del entorno, es decir, la adaptación de este con la finalidad de lograr una mayor accesibilidad cognitiva y reducir la intensidad de los estímulos sensoriales que puedan resultar molestos. Este es el proceso mediante el cual se lograría, en palabras de Paula (2015), un "autism friendly environment”. Podríamos establecer un paralelismo con los niveles de prevención comúnmente utilizados: primaria, secundaria y terciaria (Colimon, 2010, citado en Vignolo, Vacarezza, Álvarez y Sosa 2011). Este primer nivel, descrito por Paula (2015), correspondería a la prevención primaria, con la que se pretende evitar la aparición del problema o crisis. El segundo nivel haría referencia a una prevención secundaria donde ese problema está presente, con la utilización de recursos para atenuarlo o evitar que se agrave. En este punto, entrarían en juego técnicas de control de la activación emocional, técnicas de exposición y técnicas cognitivo-conductuales en casos de TEA sin discapacidad intelectual (DI). Finalmente, el tercer nivel de intervención podría asemejarse a la prevención terciaria que pretende minimizar las consecuencias del problema una vez desatado. En este caso se llevarían a cabo acciones que han de iniciarse una vez aparece la crisis (Paula, 2015).

\subsection{TEA, ansiedad y empleo}

Una vez revisada tanto la prevalencia como la etiología, evaluación e intervención en ansiedad de personas con TEA, resulta interesante dirigir la mirada a un ámbito concreto que genera niveles elevados de ansiedad en esta población: el empleo. El empleo es el puente hacia la posibilidad de desarrollar planes de vida en la edad adulta, la mayoría de las acciones a realizar en esta etapa de la vida pasan por tener un empleo remunerado, que dé acceso a aspectos de ocio o vivienda; en definitiva, acceso a un proyecto personal de vida. Las personas con TEA suelen tener mayores dificultades para acceder al mercado laboral y, sobre todo, desempeñar puestos de trabajo ordinarios. Las características propias del trastorno del que son diagnosticadas y, en muchas ocasiones, su vulnerabilidad a experimentar elevados niveles de ansiedad que se pueden afianzar como otro trastorno coocurrente, les dificulta la adquisición y/o mantenimiento de un puesto de trabajo. La primera dificultad la encuentran en los procesos de búsqueda y selección de empleo, puesto que llevan asociadas situaciones de incertidumbre: la no respuesta ante el envío de candidaturas, la dificultad de interpretar el silencio de las empresas, etc., situaciones que generan estados de ansiedad importante entre las personas con TEA en procesos de búsqueda. Otro momento complejo suelen ser las entrevistas de trabajo, donde las dificultades de interpretación del interlocutor, su lenguaje no verbal, o la literalidad en la comprensión de las preguntas y posibles respuestas a dar desencadenan miedo ante tal situación de entrevista y, por lo tanto, ansiedad. Estos procesos suelen ser la puerta de entrada hacia un empleo y, con frecuencia, no superarlos implica no acceder a un puesto de trabajo.

Por otro lado, cuando estas personas logran un determinado puesto laboral no suelen mostrar grandes dificultades en el desempeño de las tareas encomendadas, si bien manifiestan mayores dificultades a la hora de interpretar la realidad del entorno laboral, o dificultades en la comprensión de mensajes sociales o en las relaciones con los/ as compañeros/as, es decir, en aspectos muy necesarios para el mantenimiento de un puesto de trabajo. Estas dificultades generan con frecuencia mucha ansiedad porque no comprenden cómo manejar la situación, por lo que la necesidad de apoyo en este sentido se hace evidente. Necesitan apoyo para comprender las situaciones que aparecen, así como para aprender a manejar el estrés y la ansiedad que se derivan de dichos acontecimientos sociolaborales. Por ello, resulta clave intervenir sobre su estado emocional y los factores asociados con el fin de lograr un comportamiento más adaptativo a las demandas de la situación laboral. Algunos autores, como Vidriales, Hernández y Plaza (2018), proponen el entrenamiento en situaciones de procesos de selección, como la realización de entrevistas, que contribuiría a reducir la sintomatología ansiosa asociada a dichos contextos. Como indica el modelo transdiagnóstico señalado anteriormente, es importante incidir sobre tres variables: la intolerancia a la incertidumbre, la disfunción ejecutiva y la desregulación emocional (Beneytez, 2019). Esto se puede trasladar a la situación concreta de búsqueda y adquisición de empleo. Las situaciones que no son capaces de anticipar generan mayores niveles de ansiedad o estrés, igual que aquellas que valoran como negativas, muy difíciles o desagradables. Por ello, resulta esencial aconsejar y acompañar a la persona en este proceso anticipando, en la medida de lo posible, las situaciones, lo que contribuirá a reducir la incertidumbre que tan difícil les resulta de tolerar. Además, se aconseja intervenir en el plano cognitivo, trabajando sobre la atribución y valoración que hagan de los acontecimientos y tareas laborales; en el plano afectivo, donde puedan identificar cómo se sienten, qué situaciones las ponen nerviosas y qué soluciones pueden adoptarse; y en el plano 
conductual, ayudándolas a entender su propia conducta, analizando las repercusiones que tiene y cómo se podría moldear y/o modificar si no está siendo adaptativa. Todo ello teniendo en cuenta las características individuales para encontrar el momento apropiado para realizar este proceso.

\section{Objetivos del estudio}

Los principales objetivos del estudio del que se da cuenta en este artículo son:

- Evaluar los niveles de ansiedad estado (A/E) y ansiedad rasgo $(A / R)$, así como el nivel de estrés sociolaboral percibido en un grupo de adultos con TEA.

- Evaluar los niveles de ansiedad rasgo y estrés sociolaboral percibido en un grupo de contraste.

- Comparar los niveles de estrés y ansiedad, tanto como rasgo de personalidad, como aplicados a la situación laboral, por parte de los grupos seleccionados.

- Llevar a cabo el seguimiento de cada participante en el proceso con la finalidad de detectar situaciones ansiógenas y reducir dichos niveles de ansiedad y/o estrés ligados a determinadas situaciones.

\section{Hipótesis de trabajo}

Se plantea como hipótesis la relación estadísticamente significativa y positiva entre ansiedad rasgo y la percepción de estrés sociolaboral, si bien se espera una relación positiva y más significativa entre ambas variables en ambas muestras contraste, en comparación con la muestra con TEA. Esta hipótesis se plantea asumiendo la dificultad para simbolizar y anticipar o imaginar acontecimientos no experimentados por parte de los participantes con TEA. Por tanto, se esperan diferencias estadísticamente significativas entre los grupos en las puntuaciones obtenidas de la modificación de la escala de estrés sociolaboral percibido (adaptación de la escala EAE).

\section{Metodología de trabajo}

Tras la definición de los objetivos y la hipótesis de trabajo, se establece la siguiente metodología de actuación. En primer lugar, se comienza por la búsqueda y selección de instrumentos de evaluación de ansiedad y estrés en el ámbito sociolaboral. Debido a la ausencia de instrumentos adaptados y validados para adultos con TEA, se opta por la adaptación de los existentes a las características de las personas con TEA y su nivel de comprensión. De ese modo, se incluyen apoyos visuales, instrucciones e ítems más comprensibles y literales para su adecuada comprensión.
Posteriormente, se realiza una selección de posibles participantes en el proyecto por parte del equipo multidisciplinar que conforma la asociación (área de consultas y área sociolaboral de APNABI-Autismo Bizkaia). Se establece un contacto inicial con las personas seleccionadas y se propone una evaluación inicial de sus niveles de ansiedad (como estado y como rasgo de personalidad) y estrés sociolaboral. Se explica tanto de forma previa a concertar la cita, como antes de empezar la sesión de evaluación, el procedimiento de evaluación y su propósito, con el fin de fomentar su autonomía y capacidad de toma de decisiones de manera libre e informada, otorgando su consentimiento informado. Se recoge la información a través de las escalas seleccionadas respetando su intimidad y su dignidad, velando por mantener los datos personales de manera protegida y confidencial. Además, de manera paralela, se administran las escalas a un grupo de contraste a través de formularios de Google.

Finalmente, tras la recogida de datos y resultados, se lleva a cabo una nueva selección de participantes, que ya pasan a participar en el curso de búsqueda de empleo impartido por la empresa C2B (Campus to Business). La selección implica criterios como disponibilidad de horario y días por parte de la persona con TEA, motivación para realizar el curso y valoración de su necesidad en función de los resultados derivados de la evaluación emocional previa. A continuación, se adjudican los participantes a dos grupos (turno de mañana y turno de tarde), y se comienza la formación presencial. Sin embargo, debido a las circunstancias derivadas de la covid-19, el proyecto queda paralizado y se retoma dos meses después de manera telemática. El curso tiene como objetivo proveerles de herramientas para lograr una búsqueda de empleo exitosa y conseguir su inclusión sociolaboral.

Durante su participación en el curso, las personas también reciben un seguimiento individual por parte del equipo de APNABI-Autismo Bizkaia. La información se recoge a través de autorregistros, cuyo contenido aporta datos cualitativos acerca de la vivencia personal de la experiencia formativa. De manera diaria, deben completar un cuestionario donde plasman qué situaciones les han provocado estrés o ansiedad, antes o durante la clase del curso de ese día. Asimismo, también reflejan qué pensamientos y emociones han percibido, así como las acciones que han llevado a cabo para tratar de disminuir su malestar, y la eficacia de estas. De este modo, se pretende fomentar su autoconciencia, detectar posibles dificultades o situaciones incómodas y favorecer la reflexión acerca de los propios recursos de afrontamiento; a su vez, aporta información para un análisis cualitativo.

\subsection{Participantes}

Inicialmente, como se ha comentado, se realiza un proceso de selección de personas con TEA 
pertenecientes a la asociación de APNABI-Autismo Bizkaia, que cumplieran con unos ciertos requisitos de edad (>18 años), situación de desempleo o en proceso de búsqueda de este. De esa preselección se obtienen cuarenta perfiles, a los que se aplica la evaluación previa de ansiedad y estrés. De nuevo, se realiza otra selección de usuarios, escogiendo aquellos que por su perfil ansioso (los que mayor ansiedad tendían a experimentar ante procesos de búsqueda de empleo), su situación laboral y académica actual, y su disponibilidad, pueden pertenecer al grupo de los treinta elegidos para participar en el proyecto. Sin embargo, y debido a la situación provocada por la pandemia de la covid19, algunas de estas personas han tenido severas dificultades para acomodarse al nuevo formato online de formación, por lo que el grupo se ha visto reducido a veintisiete participantes con TEA.

De este modo, el estudio al que se refiere este artículo se lleva a cabo con los siguientes grupos de población:

- El primero, formado por 27 personas de APNABIAutismo Bizkaia, de las cuales 9 son mujeres y 18 hombres, de edades comprendidas entre los 18 y los 47 años, siendo la media de 25,93 años de edad.

- El segundo, formado por una muestra control de 27 personas, 14 de ellas mujeres y 13 hombres, de edades comprendidas entre los 19 y los 27 años, siendo la media de edad de 22,37.

- Los criterios de inclusión de ambos grupos en el estudio fueron:

- Ausencia de trabajo remunerado actual.

- Situación de búsqueda de empleo (o deseo de buscarlo).

- Manejo de las nuevas tecnologías para responder a los cuestionarios.

- Haber cumplido la mayoría de edad.

- Desear participar en el proyecto.

\subsection{Instrumentos}

Inicialmente se eligen dos cuestionarios para evaluar la ansiedad y el estrés en el ámbito sociolaboral en adultos. Por un lado, el Cuestionario de Ansiedad Estado-Rasgo (STAI-E y STAI-R) (Spielberg, Gorsuch y Lushene, 1988), que evalúa tanto la ansiedad ligada a una circunstancia o contexto particular, como la ansiedad en cuanto a rasgo de personalidad. Por otro lado, la Escala de Estrés Socio-Laboral (EAE-S) de la Escala de Apreciación del Estrés (EAE) (Fernández-Seara y Mielgo, 1992), que evalúa respuestas de estrés en el contexto sociolaboral. Al carecer de instrumentos que evalúen ansiedad y estrés en el ámbito sociolaboral adaptados y validados para personas adultas con TEA, se opta por realizar una adaptación de los anteriormente citados. La adaptación de estos cuestionarios incluye modificaciones tanto en el formato, de acuerdo a modelos accesibles cognitivamente (apoyos visuales, sintaxis clara y sencilla, letra grande y en mayúsculas, etc.), como en la redacción y contenido de algunos de los ítems (ajustándose a las habilidades de comprensión y al momento vital de búsqueda de empleo de las personas participantes).

Los instrumentos utilizados son aplicados en formato online, realizando así una aproximación al uso de las nuevas tecnologías que, ahora más que nunca, parece marcar el presente y futuro de la actual sociedad globalizada en tiempos de covid-19. A continuación se describe de manera pormenorizada las propiedades de cada instrumento.

El Cuestionario de Ansiedad Estado-Rasgo (STAI-E y STAI-R) (Spielberg, Gorsuch y Lushene, 1988), es un cuestionario para adolescentes y adultos, compuesto por 40 ítems. Los 20 primeros pertenecen a la escala de Ansiedad-Estado (A/E), que evalúa el estado emocional transitorio de tensión e hiperactividad del sistema nervioso autónomo (SNA), y que está ligado a la situación inmediata, es su caso, a contexto de evaluación. A partir del ítem 21 comienza la segunda escala, la de Ansiedad Rasgo (A/R), que evalúa la tendencia hacia la tensión, aprensión e hiperactividad del SNA en la mayoría de los contextos del día a día de la persona. Esta escala, por tanto, evalúa un estado más global de ansiedad, no ligado a la situación de evaluación. Por ello, se decide aplicar únicamente esta escala a la muestra de contraste, ya que el objetivo es tratar con un concepto de ansiedad más estable en el tiempo, independientemente del contexto inmediato.

Con relación a las propiedades psicométricas de este cuestionario, cabe destacar que la fiabilidad de este instrumento es bastante buena (alfa de Cronbach 0,94 en A/E y de 0,86 en A/R) en muestras de sujetos españoles normotípicos. Los índices de correlación encontrados entre las dos escalas (A/E y $A / R)$ de las que se compone el instrumento revelan que estos índices fluctúan entre 0,44 y 0,55 en mujeres, y entre 0,51 y 0,57 en hombres, es decir, es más probable que correlacione un elevado nivel de ansiedad estado con un elevado nivel de ansiedad rasgo en población masculina frente a la femenina. Por otro lado, los análisis de validez concurrente del STAI con el cuestionario de personalidad 16-PF (Cattell, 1993), la A/E correlacionada de manera estadísticamente significativa y positiva con personalidad inestable (C-), sumisa (E-), sobria $(\mathrm{F}-)$, calculadora $(\mathrm{N}+)$, aprensiva $(\mathrm{O}+)$ y frustrada (Q4+). En cuanto a las relaciones apreciadas entre $A / R$ y los factores de personalidad de dicha escala, se muestran relaciones estadísticamente significativas y positivas entre los mismos factores que correlacionan con $\mathrm{A} / \mathrm{E}$, destacando una mayor correlación positiva $(0,55-0,58)$ entre $A / R$ y Ansiedad en segundo orden.

- Adaptación de la Escala de Estrés Socio-Laboral (EAE-S) de la Escala de Apreciación del Estrés 
(EAE) (Fernández-Seara y Mielgo, 1992). La escala original está diseñada para ser aplicada a individuos de entre 18 y 85 años. Consta de cuatro escalas independientes: Escala de Estrés General (EAE-G), Escala de Acontecimientos Estresantes en Ancianos (EAE-A), Escala de Estrés Socio-Laboral (EAE-S) y Escala de Estrés en la Conducción (EAEC). Además de incluir la valoración de estrés de cada ítem, se requiere señalar si esa situación se dio en el pasado $(P)$ y con qué nivel de estrés, o si, por el contrario, sigue estando presente (A) indicando la intensidad del estrés percibido. La escala EAE-G consta de 53 ítems, la EAE-A de 51 ítems, la EAE-S contiene 50 enunciados y la EAE-C dispone 35. Los ítems de la escala EAE-S están diseñados para ser aplicados a adultos trabajadores por cuenta ajena, de edades comprendidas entre los 20 y los 60 años. Por eso no se pudo aplicar la escala original a la muestra de participantes en el proyecto, pues ni por edad ni por condición laboral cumplían los requisitos. Sin embargo, tras revisar las otras escalas, se opta por realizar una adaptación de la EAE-S, de modo que se ajustase al momento de búsqueda de empleo en el que se encuentran las personas participantes, y que estuviera redactada considerando sus habilidades, para que el formato no supusiera un obstáculo en la comprensión de la escala.

\subsection{Análisis estadísticos}

En primer lugar, cabe destacar que los niveles medios de estrés y ansiedad encontrados en el grupo con TEA (STAI-E M = 49,15; STAI-R M = 70,19; Adaptación EAE $M=51,26)$ son superiores a los reflejados por el grupo control (STAI-R M =70,19; Adaptación EAE M $=48,19)$, si bien a continuación se analiza si dicha diferencia es estadísticamente significativa.

Según los datos derivados del análisis de normalidad para cada variable, en función de la prueba de Kolmogorov-Smirnov ( $\mathrm{N}>50$ ), la distribución de la variable STAI-R es no normal, y la distribución de la variable Adaptación de EAE sigue una distribución normal en ambos grupos de población. Únicamente en el grupo con TEA la distribución de la variable STAI-E es normal. Por ello, se decide aplicar pruebas no paramétricas para analizar la variable que no sigue la distribución normal (STAI-R) y pruebas paramétricas para la que sí sigue una distribución normal en la muestra de 54 sujetos (Adaptación EAE), con relación a la diferencia de medias entre el grupo con TEA y el grupo de contraste.

Los resultados derivados del análisis no paramétrico de la U de Mann-Whitney para dos muestras independientes revelan que no existen diferencias estadísticamente significativas en la puntuación de la escala STAI-R, en función de los grupos de población (grupo con TEA y grupo contraste) $(U=257.500 ; p=$ $0,064)$. Por otro lado, el análisis paramétrico de la $t$ de Student para comparar medias en dos muestras independientes refleja que no existen tampoco diferencias estadísticamente significativas en la puntuación de la adaptación de la escala EAE en función de los grupos $(t=0,921 ; g l=52 ; p=0,361)$.

Se opta por el estadístico no paramétrico Rho de Spearman para analizar la relación entre las variables en ambos grupos de población. Se observa una relación estadísticamente significativa, positiva y moderada entre las pruebas STAI-R y la adaptación de $\operatorname{EAE}(\operatorname{Rho}=0,510 ; p=0,000)$. Al analizar dicha relación en función de los grupos de población, la correlación se muestra significativa, positiva y moderada, tanto en el grupo de personas con TEA (Rho $=0,485 ; p=0,010$ ), como en el grupo contraste (Rho $=0,507 ; p=0,007$ ).

De nuevo el estadístico no paramétrico Rho de Spearman se emplea para observar las relaciones entre las puntuaciones de las escalas STAI-R y STAI-E en el grupo de población de participantes con TEA (que únicamente se aplicó STAI-E en este grupo). Se halla una relación estadísticamente significativa, positiva y elevada entre los resultados de la escala STAI-R y STAI-E en el grupo de personas con TEA (Rho $=0,645 ; p=0,000)$.

\section{Discusión y conclusiones}

Este estudio preliminar llevado a cabo con los datos recogidos hasta el momento señala aspectos importantes a recalcar. Como se ha indicado, la ansiedad en el TEA es una condición coocurrente que parece presentarse con mayor frecuencia en este grupo frente a grupos neurotípicos (Paula, 2015). Es probable que esta relación pueda explicarse según las hipótesis planteadas en modelos transdiagnósticos, que señalan tres variables comunes a ambas condiciones: intolerancia a la incertidumbre, disfunción ejecutiva y desregulación afectiva (Beneytez, 2019). Esta condición de ansiedad que tiende a afectar a las personas con TEA se aprecia nítidamente en el contexto sociolaboral.

Acceder y mantener un empleo ordinario puede resultar ardua tarea para muchas personas adultas con TEA. Se ha de tener en cuenta que tienen severas dificultades para intuir e interpretar conceptos simbólicos y abstractos, para predecir y anticipar las consecuencias de acciones propias y ajenas en terceras personas, para ajustarse emocionalmente a las exigencias del entorno o para mantener relaciones sociales recíprocas. Todo esto relacionado con conceptos tan relevantes como el de ToM, la pragmática del lenguaje y/o la alexitimia (Paula, 2015).

Los datos derivados de la evaluación de los niveles de ansiedad y estrés en el ámbito sociolaboral de los participantes en el estudio del que se da cuenta en este artículo muestran que las personas adultas con TEA tienen niveles de ansiedad y estrés más elevados que el grupo de población de contraste, si bien dicha 
diferencia no es estadísticamente significativa. A pesar de encontrar una muestra significativa de personas participantes dentro de la entidad ( $\mathrm{N}$ $=27$ ), la inexistencia de pruebas psicométricas validadas que puedan evaluar la ansiedad y el estrés en personas con TEA supone una limitación. Las escalas aplicadas con su respectiva modificación es posible que no tengan la validez suficiente como para poder extraer de ellas resultados concluyentes en personas adultas con TEA. Como se ha citado anteriormente, las personas con TEA tienden a tener dificultades para reconocer y expresar sus propias experiencias emocionales, lo que lleva a plantear si constituyen una medición válida y representativa de su realidad, si realmente ofrecen puntuaciones inferiores a lo que personas neurotípicas exhibirían en su misma situación (vivenciando los mismos niveles de estrés y ansiedad) o tales puntuaciones se hallan condicionadas por sus dificultades. Al ser una puntuación subjetiva, muy influenciada por la capacidad de insight de cada individuo, es posible que se encuentren sesgos en esta población diana. Este es un dato fundamental a tener en cuenta en el manejo de situaciones ansiógenas con personas con TEA. Conociendo al colectivo y considerando sus severas dificultades en el mencionado plano psicosocial y emocional, tanto a nivel interpersonal como intrapersonal, se ha de contemplar la posibilidad de que los resultados extraídos de las evaluaciones en ansiedad y estrés puedan estar enmascarando su realidad.

A nivel cualitativo, gracias al proceso de seguimiento de los niveles de ansiedad y estrés durante la impartición del curso, se recoge cómo algunas situaciones cotidianas como "problemas para conectarme al curso (mal funcionamiento de Internet)" o "escuchar a mis compañeros" o "participar hablando en el curso", les generan malestar y pueden llegar a identificarlo en síntomas fisiológicos (sensaciones físicas) y pensamientos. Las situaciones más señaladas como estresores son: "pensar que tengo que participar en el curso (hablando)" y "participar hablando en el curso". Los síntomas fisiológicos más comunes, según la mayoría de los participantes con TEA que han reflejado sentir ansiedad, son: "voz entrecortada (dificultades para hablar de manera fluida)", “taquicardias" y “dolor de tripa”. Por su parte, los pensamientos más recurrentes son: "que no voy a conseguir hablar/expresarme como yo quiero", “que los demás no me van a entender" y "que no valgo para esto". Y, finalmente, los recursos de acción que acometen con el fin de intentar reducir esa tensión o estrés suelen ser: "escribir en un papel lo que voy a decir para ensayarlo antes”, "pensar que sí voy a ser capaz" y “controlar mi respiración (respirar más despacio y profundamente)". Sin embargo, la gran mayoría evalúa estos planes de acción como poco efectivos, pues refieren que reducen poco o nada su estado de nerviosismo. Por tanto, estos indicadores abren una puerta hacia el desarrollo de planes de intervención que incluyan el entrenamiento en el manejo de situaciones ansiógenas. Según los datos aportados por las personas con TEA participantes en este proyecto, se puede indicar que la mayoría refleja no tener las herramientas o recursos suficientes 0 , mejor dicho, efectivos, para reducir su malestar.

En estos momentos el proyecto continúa en marcha, por lo que se plantean nuevos objetivos a desarrollar a corto-medio plazo:

- Lograr una muestra de contraste con un número de hombres superior (hombres de la muestra de contraste $48,15 \%$ frente al de hombre de la muestra con TEA 66,67\%), de modo que se equipare al porcentaje encontrado entre las personas con TEA. Este aumento del número de hombres de la muestra de contraste se considera la mejor alternativa de cara a equiparar las muestras en cuanto a género, dado que existe en el colectivo de personas con TEA una prevalencia de 1:4 (1 mujer por cada 4 hombres). Esta es una línea de investigación actual, ya que se desconoce si es real la desproporción a causa del TEA o debido al enmascaramiento de los síntomas en el caso de las mujeres (Confederación Autismo España).

- Realizar una evaluación de los niveles de ansiedad y estrés en el ámbito sociolaboral tras haber finalizado la formación y el seguimiento, y observar si existen cambios.

- Trabajar con las personas con TEA técnicas que puedan implementarse en los entornos naturales donde participan, y que les permitan manejar de manera más exitosa sus estados de tensión y su malestar.

Desde APNABI-Autismo Bizkaia se abre la puerta a futuras colaboraciones, ya que para determinar si los instrumentos modificados y adaptados pudieran ser válidos y fiables para aplicar a adultos con TEA, se requeriría de un tamaño muestral superior al disponible por el momento.

Finalmente, cabe concluir que desde este proyecto, al igual que desde todos los servicios que ofrece la asociación, se aboga por mejorar la calidad de vida de las personas con TEA, fomentando su autonomía y su inclusión en la sociedad como ciudadanos/as de pleno derecho, donde poder desarrollar de forma satisfactoria sus planes de vida. 


\section{Referencias bibliográficas}

AMERICAN PSYCHIATRIC ASSOCIATION (2013): Diagnostic and statistical manual of mental disorders, Washington DC, APA.

BENEYTEZ, C. (2019): "Síntomas de ansiedad en los trastornos del espectro del autismo desde un enfoque transdiagnóstico" [tesis doctoral], Madrid, Universidad Complutense de Madrid, 〈https://eprints.ucm.es/56729/〉.

CATTELL, R. B.; CATTELL, A. K.; y CATTELL, H.E.P. (1993): Sixteen Personality Factor Questionnaire, Institute for Personality and Ability Testing, Inc.

CLARK, D.A.; y BECK, A.T. (2012): Terapia cognitiva para trastornos de ansiedad, Serie Psicoterapias Cognitivas, Bilbao, Desclée de Brouwer.

CONFEDERACIÓN AUTISMO ESPAÑA: "Sobre el TEA", 〈http:// www.autismo.org.es/sobre-los-TEA/trastornodel-especto-del-autismo>.

CRESPO, M. y LABRADOR, F.J. (2003): Estrés, Serie Guías de Intervención, Madrid, Síntesis.

FERNÁNDEZ-SEARA, J.L. y MIELGO, M. (1992): EAE: Escalas de Apreciación del Estrés, Madrid, TEA Ediciones.

HERVÁS, A. (2016): “Un autismo, varios autismos. Variabilidad fenotípica en los trastornos del espectro autista". Revista de Neurología, vol. 62, no 1, pp. 9-14. 〈https://doi.org/10.33588/ rn.62S01.2016068>.

IVERN, I. (2017): "Pragmática y desarrollo metalingüístico", Revista de Logopedia, Foniatría y Audiología, vol. 38, no 1, pp. 31-39. < https://doi. org/10.1016/j.rlfa.2017.12.003〉.

PAULA, I. (2013): “Coocurrencia entre ansiedad y autismo. La hipótesis del error social y de la carga alostática”, Revista de Neurología, vol. 56, no 1, pp. 45-59. 〈https://doi.org/10.33588/ rn.56S01.2012652>.

- (2015): La ansiedad en el autismo, Madrid, Alianza Editorial.

POSTORINO, V.; KERNS, C.M.; VIVANTI, G.; BRADSHAW, J.; SIRACUSANO, M. y MAZZONE, L. (2017):
"Anxiety Disorders and Obsessive-Compulsive Disorder in Individuals with Autism Spectrum Disorder", Current Psychiatry Reports, vol. 19, no ${ }^{12}$, < https://doi.org/10.1007/s11920-0170846-y>.

SÁNCHEZ, M.S.; ALONSO, Y.; y ALCANTUD, F. (2018): “Análisis de las estrategias de evaluación de la ansiedad en niños/as con Trastorno del Espectro del Autismo (TEA)", En MOLERO, M.M.; PÉREZ-FUENTES, M.C.; GÁZQUEZ, J.J.; SIMÓN, M.M.; BARRAGÁN, A.B.; MARTOS, A.; y SISTO, M. (ed.), Intervención en contextos clínicos y de la salud, España, pp. 89-96.

SPIELBERG, C.D.; GORSUCH, R.L. y LUSHENE, R. (1982): Manual del Cuestionario de Ansiedad Estado/ Rasgo (STAI), Madrid, TEA Ediciones.

TIRAPU-USTÁRROZ, J.; PÉREZ-SAYES, G.; EREKATXO-BILBAO, M. y PELEGRÍN-VALERO, C. (2007): “¿Qué es la Teoría de la Mente?", Revista de Neurología, vol. 44, no 8, pp. 479-489. 〈https://doi. org/10.33588/rn.4408.2006295>.

VAN STEENSEL, F.J.A.; BÖGELS, S.M. y PERRIN, S. (2011): "Anxiety disorders in children and adolescents with autistic spectrum disorders: a metaanalysis", Clinical Child and Family Psychology Review, vol. 14, n- 3, pp. 302-317, < https://doi. org/10.1007/s10567-011-0097-0>.

VIDRIALES, R.; HERNÁNDEZ, C. y PLAZA, M. (2018): Empleo y Trastorno del Espectro del Autismo: "Un potencial por descubrir", Madrid, Autismo España, <http://www.autismo.org.es/sites/ default/files/un_potencial_por_descubrir_ digital_baja_cuerpo_junio_o.pdf $\rangle$.

VIGNOLO, J.; VACAREZZA, M.; ÁLVAREZ, C. y SOSA, A. (2011): "Niveles de atención, de prevención y de atención primaria de la salud", Archivos de medicina interna, vol. 33, $\mathrm{n}$ - 1, pp. 11-14, 〈http://www.scielo.edu.uy/pdf/ami/v33n1/ v33n1a03.pdf $\rangle$. 